\title{
Rénovation urbaine et stratégies familiales : une étude de cas
}

\author{
In: Sociétés contemporaines N¹7, Mars 1994. pp. 11-27.
}

\begin{abstract}
Based on the results of a recent anthropological study, this paper analyzes the effects of an inner-city restoration on the families who were living there before. Although they went through a long economic impoverishment process, some of them were able to develop social strategies which were linked with their strong territorial insertion, and consisted in a socio-cultural asset mobilisation. This urban policy allowed them to convert their locally useful assets into a more widely valuable currency. At the same time they created new career opportunities for themselves.
\end{abstract}

\section{Résumé}

Cet article analyse les effets d'une opération de rénovation urbaine, menée dans un quartier urbain central d'une ville moyenne, sur la population qui en était originaire. Il s'agit d'une population traditionnellement ouvrière qui, à travers plusieurs facteurs liés à I'histoire de l' agglomération, s'était trouvée paupérisée. Bien que de faible niveau socio-économique, certains ménages ont pu mettre en place des strategies sociales efficaces à partir d'une mobilisation de ressources culturelles liées à leur forte insertion territoriale. Cette mobilisation semble s'orienter vers une reconversion d' un capital essentiellement à usage local en un capital à valeur plus générale. La rénovation aura pu ainsi constituer un opérateur de déblocage de trajectoires sociales.

Citer ce document / Cite this document :

Bidou-Zachariasen Catherine. Rénovation urbaine et stratégies familiales : une étude de cas. In: Sociétés contemporaines No17, Mars 1994. pp. 11-27.

doi : $10.3406 /$ socco.1994.1152

http://www.persee.fr/web/revues/home/prescript/article/socco_1150-1944_1994_num_17_1_1152 


\title{
RÉNOVATION URBAINE ET STRATÉGIES FAMILIALES : UNE ÉTUDE DE CAS ${ }^{1}$
}

\begin{abstract}
Rtsumt : Cet article analyse les effets d'une opération de rénovation urbaine, menée dans un quartier urbain central d'une ville moyenne, sur la population qui en était originaire. Il s'agit d'une population traditionnellement ouvrière qui, à travers plusieurs facteurs liés à l' histoire de l'agglomération, s'était trouvée paupérisée. Bien que de faible niveau socio-économique, certains ménages ont pu mettre en place des stratégies sociales efficaces à partir d'une mobilisation de ressources culturelles liées à leur forte insertion territoriale. Cette mobilisation semble s'orienter vers une reconversion d' un capital essentiellement à usage local en un capital à valeur plus générale. La rénovation aura pu ainsi constituer un opéraleur de déblocage de trajectoires sociales.
\end{abstract}

"La rénovation ne bouscule pas sculement des murs et des rues, elle affecte profondément un groupe humain, provoque ou accélère une mutation des structures locales et sociales; ses effets dépassent singulièrement le seul domaine de l'habitat et doivent s'étudier comme une forme particulière du changement social en général. » constatait l'auteur d'une recherche sur les transformations sociales qu'avait connues une partie du treizième arrondissement de Paris après avoir été rénové (Coing, 1966). Il notait également que la conjoncture de rénovation urbaine représentait une situation expérimentale intéressante car facile à constituer en variable indépendante. Dans le cas qui va nous concerner, une opération de rénovation d'un quartier central, populaire et dégradé d'une ville de moyenne importance, la situation sera d'autant mieux cernće que la proportion de population demeurée dans le quartier après rénovation sera relativement importante par rapport à ce qui se passe couramment dans ce type d'opération ${ }^{2}$. Cependant ce qui nous intéressera ici ne sera pas tant le changement social en général dans le quartier que les effets de l'événement " rénovation » sur cette partie de l'ancienne population qui a fait le « choix » ou retenu l'option de rester dans le quartier ${ }^{3}$.

I. Je remercie Edmond Preteceille qui m'a permis par ses remarques d'améliorer la première version de ce texte.

2. Plus de la moitié restera ici dans le quartier rénové contre à peine plus d'un quart dans le cas de l'îlot Jeanne d'A rc, étudié par Coing (1966).

3. Nous utiliserons ici les données recueillies lors d'une recherche menée pour le compte de la Mission du Patrimoine Ethnologique du Ministère de la Culture, qui portait sur cette rénovation envisagée à partir d'une double perspective, d'une part celle des politiques locales, en particulier celles concemant 
À partir d'une analyse du contexte socio-territorial nous allons étudier comment des acteurs sociaux ont subi un événement (l'opération urbaine) qui dura plusieurs années, ou s'en sont emparés, ou les deux à la fois. Qu'est ce qui s'est trouvé engagé dans l'accès au nouveau logement, comment a été négociée une sorte de synthèse entre anciennes et nouvelles pratiques quotidiennes, entre anciens et nouveaux modes d'exercices de citoyenneté locale ? Mais nous verrons comment, au delà des évolutions formelles qui ont marqué les vies matérielles, c'est beaucoup plus qui s'est joué. Bien que la saisie du processus pose problème en raison du peu de recul temporel, les ménages (et non pas les individus c'est à dire déjà une résultante de rapport de forces entre des individus) semblent avoir été en mesure, pas tous avec la même réussite ni la même aisance, de développer des stratégies sociales, qu'on pourrait définir comme de rétablissement ou de déblocage de trajectoires. Nous allons montrer comment, à partir de l'opportunité de l'événement rénovation, il y a eu mobilisation de différents types de ressources et tentatives de reconversion du capital que celles-ci représentaient dans le contexte socio-territorial antérieur en un capital utilisable dans le nouveau contexte.

\section{UN CONTEXTE SOCIO-TERRITORIAL}

Au nord de la cathédrale d'Amiens le quartier de Saint-Leu s'étend sur une vingtaine d'hectares. Sa trame urbaine est marquée par un réseau de bras de rivières et canaux qui furent à l'origine d'implantations industrielles (moulins, teintureries, filatures) qui demeurèrent prospères des siècles durant. Le quartier abritait alors toutes les catégories de population, ouvrières, commerçantes voire bourgeoises. Mais dès la fin du dix-neuvième siècle il connut une première vague de désindustrialisation et perdit aussi son caractère de population mélangée. Seule la main d'ocuvre locale continua à y résider. Dans les années mille-neuf-cent-cinquante quelques entreprises étaient encore en activité et la population, essentiellement ouvrière, travaillant ou non sur place, avait conservé une grande stabilité résidentielle en dépit d'un habitat en mauvais état. Mais son faible niveau socio-ćconomique et l'insalubrité du cadre de vie avaient cristallisé autour de Saint-Leu une très mauvaise image.

Les multiples projets de rénovation élaborés par les Municipalités successives n'imaginèrent pas d'autre solution que son arasement. La construction des ZUP et

\footnotetext{
la « valorisation culturelle » du centre-ville, et d'autre part celle des transformations sociales engagées par ces mêmes politiques (Bidou-Zachariasen, 1993). Au point de vue méthodologique, le volet portant sur le quartier et ses habitants a été mené selon une approche de type anthropologie urbaine classique mêlant l'analyse de documents et de statistiques, l'observation de pratiques et l'analyse d'entretiens semi-directifs. Ceux-ci ont été menés en deux étapes auprès d'un échantillon d'une cinquantaine de ménages (dont trente-cinq originaires du quartier et quinze nouvellement arrivés). Pour les ménages « originaires », qui seuls concernent le présent article, les premiers entretiens ont porté sur le déménagement, le nouveau logement et le nouveau quarier comparés aux précédents, l'impact du changement sur leurs organisations de vie quotidiennes. Dans un deuxième entretien nous les avons interrogés très longuement sur leurs histoires personnelles et familiales (dans le quartier et an général). En leur faisant ainsi remonter le temps on a essayé de reconstituer avec eux leurs trajectoires sociales et familiales. Les interviews ont été complétés par des questionnaires fermés portant entre autres sur : la structure du ménage, le réseau familial (dans et hors quartier), l'appanenance associative, les emplois (y compris ascendants et enfants adultes), les niveaux scolaires, les salaires, les revenus globaux, les loyers ou traites, les charges fixes.
} 
de la zone industrielle dans les années soixante vidèrent le quartier d'une partie de ses forces vives qui préférèrent le confort d'un habitat moderne tandis que les dernières entreprises quittèrent des locaux vétustes pour se redéployer en périphérie. D'autre part les transformations et la modernisation qui avaient marqué la ville s'étaient traduites aussi par la destruction de tout un habitat précaire tandis que dans le quartier les Municipalités, depuis un certain temps déjà, procédaient au rachat des maisons délaissées afin de se constituer en maître d'ouvrage unique de la future rénovation. Dans cet habitat délabré mais vacant elles logèrent, de façon en principe temporaire, les ménages chassés par ces éradications et qui n'avaient pas les ressources suffisantes pour payer des loyers de HLM.

\section{LES CARACTERISTIQUES SOCIO-DÉMOGRAPHIQUES DU QUARTIER \\ DANS SON ENVIRONNEMENT URBAIN AVANT LA RENOVATION}

Alors que dans les années cinquante le quartier comptait dans les 3500 habitants, il va connaître une décroissance constante jusqu'aux premières années de la mise en oeuvre de sa rénovation, 2600 habitants au recensement de 1975, et 2200 à celui de 1982. Sa structure démographique ne subit pour autant de transformations profondes. Si c'est surtout des jeunes adultes qui le quittèrent pour trouver ailleurs en particulier dans les ZUP périphériques récentes de meilleures conditions de logement, les ménages « précaires » plus tardivement arrivés appartenaient aux mêmes catégories d'âge. Par rapport aux quartiers centraux limitrophes, Saint-Lcu était démographiquement plus équilibré, avec plus d'enfants $(30 \%$ de moins de vingt ans pour seulement $18 \%$ dans le centre et plus aussi de jeunes adultes, mais des personnes âgées en moindre proportion. Par rapport aux ZUP par contre, il comptait plus de personnes âgées et moins d'enfants. Les ménages étrangers étaient dans ce quartier plus nombreux que dans la moyenne d'Amiens (8,5\% - surtout portugais - contre 5,5\%) mais deux fois moins nombreux que dans les ZUP.

Les caractéristiques socio-économiques de Saint-Leu individualisaient également ce quartier par rapport aux autres quartiers centraux (Debonneuil, Golac, 1978).

En 1975 la population active comptait $60 \%$ d'ouvriers et personnels de service et $15 \%$ d'employés. En 1982, plus d'employés et moins d'ouvriers mais toujours plus de $70 \%$ de catégories populaires. Par contre les cadres supéricurs et moyens cumulés ne représentaient que $11 \%$ de la population active en 1975 comme en 1982, alors que dans le centre ceux-ci aux mêmes dates étaient plus de $45 \%$ et les catégories populaires, ouvriers et employés sculement $10 \%$.

La population active féminine représentait $30 \%$ de la population féminine totale, soit un peu moins que la moyenne pour la ville (37\%).

Les chômeurs étaient à Saint-Leu plus nombreux que partout ailleurs dans l'agglomération : $20 \%$ en 1982 contre $11,5 \%$ pour l'ensemble de la ville, $16 \%$ dans les quartiers de type ZUP et seulement $7 \%$ dans les quartiers hyper-centraux. Les revenus déclarés du travail étaient particulièrement bas, plus de la moitié des ménages gagnant moins que le SMIG.

Sources : Recensements INSEE, 1975, 1982 ; Enquête Municipale, 1976. 
Parmi les plus âgés des habitants que nous avons rencontrés, nombreux étaient ceux qui avaient été ouvriers et ouvrières du textile. Cette forte correspondance entre un territoire de travail et de résidence est sans doute au principe de cette micro-société locale qui avait sédimenté au cours des siècles dans une triple composante, ouvrière, urbaine et picarde. La disparition progressive de l'activité économique ne semblait pas avoir remis en cause l'identité territoriale que les habitants ont continué à partager et qui s'alimentait encore de sa spécificité morphologique. L'étroitesse des ruelles et canaux que bordaient des rangées de minuscules maisons distinguait en effet Saint-Leu du tissu urbain environnant, comme l'avait illustré une précédente étude (Plan Construction, 1979). Cet habitat était corrélatif de modes de vie et d'un rapport à l'espace particuliers, au sens où Lefebvre (1968) utilisait ces notions. Les logements de petite taille et sans espace intermédiaire entre public et privé, étaient, lorsque le temps le permettait, ouverts en permanence sur 1' " espace-rue » qui fonctionnait de fait comme espace de médiation. Il n'était pas perçu comme espace public mais plutôt comme espace communautaire. Appropriable et approprié par les habitants et le voisinage, il pouvait tout à la fois être la scène de conversations continuelles, des jeux d'enfants, parfois même de repas. Ce rapport à l'espace n'était pas spécifique au quartier, il était celui des habitants des quarticrs ouvriers traditionnels (Verret, 1979). Il s'était seulement prolongé ici plus longtemps qu'ailleurs.

Un des fondements de cette " société de quartier » qui jusqu'à la veille de la rénovation se vivait comme « village » et dont le mode de sociabilité dominante était la « communauté » (Gurvitch, 1963), résidait aussi dans le réseau de parenté locale qui s'y était constitué et reproduit depuis des générations et en cela elle était très proche de ce quartier du sud-est londonien étudié dans les années cinquante par Willmott et Young (1957) et dont ils écrivaient : "Bethnal Green n'est pas le refuge provisoire d'individus atomisés par l'industrie et la grande ville, mais une communauté où les relations de parenté sont un aspect essentiels de la vie quotidienne ». A Saint-Leu presque tous les habitants étaient d'une manière ou d'une autre parents ou alliés entre cux, à l'intéricur d'un nombre restreint de "grandes familles », et ce réseau était complexifié par les fréquentes unions successives et enfants dits de « lits différents ». Lorsqu'on a interrogé les plus âgés sur leurs anciens réseaux locaux d'interconnaissances et d'interfréquentations, on a pu constater leur importance et leur complexité. Chacun des interviewés connaissait à peu près toute la communauté de voisinage, avait des relations avec un grand nombre de ses membres. Par contre ces réscaux sociaux semblaient s'arrêter à la frontière géographique du quartier. Ce tissu social serré avait sans doute permis que se développe et perdure ici aussi plus qu'ailleurs une solidarité quasi-institutionnalisée qui amortissait les effets de la pauvreté et qui avait perduré jusqu'à ces toutes dernières années. S'il faut prendre garde de faire la part des choses lorsque l'on écoute des discours qui ne sont que des reconstitutions parfois mythiques du passé, on peut sans doute considérer comme véridique une certaine qualité du lien social, malgré ou plutôt au delà des problèmes sociaux liés à la grande pauvreté, comme des pratiques d'alcoolisme, qui étaient aussi une des dimensions constituantes de l'identité locale.

Mais au delà de ces particularités, telles la forme et la structure de ses réseaux sociaux qui pouvaient faire apparaître le quartier comme refermé sur lui-même, selon 
d'autres critères il pouvait au contraire être perçu comme particulièrement ouvert, par exemple vis à vis de la " chose publique ». Il se démarquait encore en cela par rapport aux modalités de citoyennetés urbaines des quartiers centraux limitrophes, ou même d'autres quartiers urbains populaires. C'était tout d'abord dans le militantisme politique que cette citoyenneté s'exprimait. La cellule locale du Parti Communiste y était particulièrement active et regroupait une partic importante de la population. La citoyenneté locale se manifestait aussi depuis la fin du siècle dernier à travers un vie associative dynamique qui couvrait une pluralité de domaines, depuis des activités culturelles traditionnelles, comme le théâtre de marionnettes, jusqu'à d'autres à caractère plus social. Le sport encore constituait un domaine d'investissement associatif important. Dans les dernières décennies c'est sur le football que les énergies locales s'étaient le plus mobilisées. Les matchs du dimanche constituaient le prétexte à des sorties en groupe, en famille, et à des manifestations parfois bruyantes de soutien aux siens, particulièrement lorsqu'ils se passaient « à domicile ». Le terrain de « foot », contigu au quartier, et sa buvette offraient en pleine ville un licu de retrouvailles quasi-campagnard. L'exacerbation du " nous » qui semblait être l'enjeu de ces loisirs populaires, tant dans le soutien à l'équipe locale que dans l'usage exclusif de l'espace, évoquait la scénographie qui organisait les dimanches des couches populaires anglaises dans l'immédiat après-guerre (Hoggart, 1970).

Bien que perçu en général de l'extérieur comme une entité sociale homogène, le quartier n'en était pas moins, comme tout « village », géographiquement et socialement stratifié. On pouvait y distinguer, et les habitants eux-mêmes distinguaient, trois sous-groupes dont la hićrarchie semblait correspondre à l'ancienneté d'installation familiale dans le quartier. Au sommet un petit groupe se vivait comme une sorte de petite bourgeoisie d'origine ouvrière et regroupait les plus anciennes familles liées au travail du textile. Ce sous-groupe correspondait à des familles qui au-delà de la disparition des métiers avaient déjà négocié des trajectoires d'ascension sociale. Celles-ci s'étaient accomplies essentiellement à travers l'accession à la propriété et la reconversion professionnelle réussie. On y trouvait des artisans, commerçants, petits entrepreneurs. Ils résidaient dans la partie sud du quartier qui correspondait originairement à sa partie portuaire et commerçante. Leurs enfants, en mobilité ascendante, avaient en général quitté le quartier. Un second sous-groupe d' "anciens » habitants était constitué d'une population un peu moins liée à l'activité traditionnelle du quartier, d'âge plus varié, car en faible mobilité sociale. Parents, enfants mariés, grands-parents, les familles évoluaient et se recomposaient à proximité les unes des autres. Beaucoup avaient des emplois liés au secteur public, Etat ou Ville. Il a déjà été montré ailleurs comment des réseaux familiaux intriqués aux affiliations politiques peuvent constituer des modes d'accès efficaces à des emplois publics locaux (Segalen, 1990). On trouve ici des mêmes types d'usages sociaux de la parenté. C'est surtout autour de la place centrale que ce « noyau dur » du quartier résidait. Les locataires y étaient majoritaires et les familles pouvaient changer fréquemment de maisons en fonction de leur agrandissement ou au contraire du départ des enfants. Elles n'allaient cependant jamais très loin et les nouveaux emménagements se réalisaient à l'intéricur d'un périmètre restreint. Ce sous-ensemble n'était d'ailleurs pas réellement distinct du précédent et l'on trouvait entre eux quelques relations familiales et d'alliance. La troisième composante concernait les ménages les plus récemment arrivés, logés par les Municipalités 
successives dans un parc immobilier constitué en vue de la future rénovation, parc fortement dégradé et dans lequel ceux-ci habitaient souvent à titre gratuit ou en payant un loyer symbolique. Pcu intégrés au travail salarié, leurs revenus, outre les prestations sociales, provenaient d'activités au noir de type brocante ou ferraille, parfois de revenus moins " légitimes » et la « débrouille » était chez eux érigée en principe de vie, comme une enquête antérieure l'avait montré (Sclim, 1982). Coutumières de l'exclusion géographique et sociale en raison de leur marginalité économique, ces familles s'étaient à leur manière intégrées au quartier. Cette intégration s'était surtout réalisée à travers un certain nombre d'alliances contractées par leurs enfants parmi la population d'origine. Ces ménages ont le plus souvent été logés dans les rues nord du quartier, où les maisons étaient les plus dégradées. Certains néanmoins résidaient au milieu des familles plus traditionnelles et des liens de voisinage et même d'entraide s'étaient établis. Ces trois sous-groupes sociaux étaient donc en recouvrement partiel et une certaine mobilité pouvait exister de l'un à l'autre.

Cependant malgré les aspects positifs qu'avait longtemps présenté ce territoire pour ses habitants, tant d'un point de vue matériel (entraide) que symbolique, offrant des éléments compensatoires d'identification à ceux qui étaient en perte d'identité professionnelle, il avait aussi pu être à l'origine de lourdes dérives individuelles. À étudier les histoires de familles on a pu constater la grave paupérisation qui a marqué beaucoup d'entre elles dans cette période charnière de la fin des années soixante et années soixante-dix, période qui a correspondu à la fois à un renouvellement démographique local, à la fermeture des dernières entreprises et au début de la montée générale du chômage. Etant donné le petit nombre de "grandes familles » concernées par le réseau local de parenté, on a pu assez. bien reconstituer les histoires croisées de chacune d'entre elles, les évolutions et les étapes de leurs différents membres. Certains à cette époque ont apparemment fait un choix d'éloignement du quartier pour fuir sa pesantcur sociale, d'autres n'eurent pas les moyens de ce type de choix et ont pu s'être trouvés enfermés dans une véritable « culture de la pauvreté ». Le quartier avait offert des ressources pour vivre avec ou malgré la pauvreté mais pas les recettes pour en sortir ${ }^{4}$. C'est ce que l'on saisit à travers les récits de trajectoires compliquées et parfois semées d'événements dramatiques.

\section{UNE OPÉRATION URBAINE INNOVANTE}

Lorsque les élections municipales de 1971 portèrent au pouvoir un nouveau maire, institutcur communiste, l'insalubrité et la paupérisation du quartier étaient très accentuées. Enfermée dans le périmètre de ses ruelles et canaux, la population y vivait comme retranchée dans la crainte de sa propre disparition. Malgré cela le « mythe » de Saint-Leu, à la fois mauvais quartier mais vivant et " authentique » avait attiré un certain nombre de jeunes, d'étudiants, d'artistes qui s'étaient mis à y louer ou même acheter des vieilles maisons. Toute une " mouvance " militante de type alternatif $s^{\prime} y$ était aussi développée dans ces années-là. La nouvelle équipe entama, ainsi qu'elle l'avait annoncé dans sa campagne électorale, les études nécessaires

4. Dans une étude portant sur les villes anglaises l'auteur estime utile pour analyser la dégradation de certains quartiers de centres anciens, le recours à la notion tant décriée de « culture de la pauvreté ». Nous avons ici la même position. (Harrison, 1983). 
à l'élaboration d'un programme de rénovation. Celui-ci devait reposer sur quelques principes de base, la préservation des caractéristiques architecturales et urbanistiques et l'offre du maintien sur place pour la population. Il s'agissait de mêler la « réhabilitation » des maisons dont l'état l'autoriserait et la « rénovation » par reconstruction de l'habitat trop dégradé selon une architecture modernisée mais qui en respecterait l'esprit. Une légère densification du bâti devait engager un processus de repeuplement ( 3500 à 4000 habitants envisagés en fin de rénovation). Si l'opération était déjà complexe à mener à bien pour des raisons particulières - surcoûts des travaux en raison de l'humidité du site - la logique générale d'évolution des centres-villes qui était à leur «gentryfication » conférait à la solution retenue un caractère déphasé qui rendit difficile son montage financier. Cependant les acteurs locaux surent utiliser au coup par coup un certain nombre de dispositifs techniques offerts au cours des années par l'arsenal des politiques de l'habitat (voir encadré).

À la fin de la décennie quatre-vingt, l'espace public, rues, ruclles, canaux, ponts et passerelles, comme la majorité des habitations, avaient retrouvé un aspect pimpant. Des nouveaux ensembles de logements à deux ou trois niveaux alternaient avec des maisons anciennes complètement refaites parfois agrandies. Les abords du quartier avaient aussi été rénovés, le désenclavant par rapport au tissu urbain environnant. Malgré un certain degré de renouvellement de la population, les relogements sur place d'anciens ménages ont été nombreux, et différentiellement localisés. La partie sud du quartier, la plus proche de la cathédrale et la plus exposée à d'éventuelles visites de touristes, a été celle qui a connu la plus grande transformation sociologique. Les anciens petits proprićtaires y demeuraient minoritaires. Beaucoup de maisons ont été vendues et les anciens commerces se sont transformés en restaurants ou boutiques peu intégrés à l'ancien quartier. Toute la partie centrale où résidaient ceux que l'on a dénommés plus haut le « noyau dur » a gardé beaucoup de ses anciens habitants. C'est là qu'on été construits le plus de logements neufs, petits ensembles HLM, majoritairement habités par des familles " originaires ». La place centrale, centre géographique et social du quartier, et les rues qui l'entourent ont conservé leurs maisons anciennes et quelques nouveaux résidents sont venus s'intercaler au milieux des anciennes familles. Dans tout le nord, encore en attente de rénovation, demeuraient à la veille des élections municipales de 1989 des ménages aux ressources économiques très faibles, progressivement repoussés par la rénovation des autres secteurs.

Au moment de l'enquête (1991) la Municipalité issue de urnes de 1989 (maire UDF) mettait en vente sur le marché le reste du parc non rénové appartenant à la Ville ou à l'Office HLM, avec propositions de relogements en parc social périphérique. La nouvelle équipe avait également peu après son arrivée entrepris la construction d'une université, dans la partie oucst du quartier, sur un vaste terrain laissé vacant par la destruction quelques années auparavant d'une grande brasserie. Ce projet était déjà celui de l'ancienne équipe ; il a été repris et activé à la faveur du plan Université 2000 lancé par le gouvernement. Depuis l'ouverture de l'université de Picardie-Jules Verne de Saint-Leu, à la rentrée d'octobre 1992, des cafés ont ouvert, des maisons réhabilitées sont habitécs par des enseignants et étudiants, évolution mieux acceptée par les anciens habitants que la tendance antérieure qui ouvrait le quartier au tourisme. 


\section{LA RENOVATION, OUTILS ET RESULTATS}

La Ville a eu recours tout d'abord à des crédits du FAU (fonds d'aménagement urbain) pour ses premières études. Puis elle a pu profiter de certaines procédures issues de la réforme de la politique du logement de 1977 comme les OPAH (opérations programmées d'améliorations de l'habitat) qui permettaient aux petits propriétaires, résidents ou bailleurs d'obtenir des subventions pour travaux qui ouvrait le droit aux APL (aide personnalisée au logement). Les logements neufs furent construits par deux offices HLM locaux dans le cadre des PLA (prêts locatifs aidés) ouvrant aussi droit aux APL, plus élevées que les anciennes «allocations-logement ». En 1984 la Ville candidata et obtint pour Saint-Leu une procédure DSQ (développement social des quartiers) qui durant cinq ans, à travers une structure opérationnelle permanente et des crédits plus réguliers, améliora le déroulement del'opération. Outre les crédits concernant le bâti, cette structure assura aussi l'encadrement social des relogements.

Au cours des années cependant, le maître d'ouvrage prenant conscience que les opérations menées en PLA et d'un niveau de prestations assez élevé, et donc à loyers et charges relativement élevés, risquaient d'exclure un certain nombre de ménages, comme ceux qui n'avaient pas assez d'enfants pour prétendre à des APL consóquentes, ou ceux dont les enfants atteignaient leur majorité. Il diversifia les formules de financement et utilisa des crédits PALULOS (prime à l'amćlioration des logements à occupation sociale) qui exigeaient des normes de confort moins élevées et donc de moindre coût pour les habitants (pour une analyse de ces procédures voir Blanc, 1990).

C'est aussi une formule originale d'auto-réhabilitation de leur propre maison qui fut offerte à des locataires, avec option d'accession à la proprićté ou même location-vente. Encadrés et outillés par les techniciens d'une association de Castors, ceux qui se sentaient les capacités de mener à bien les travaux de gros ocuvre purent ainsi accéder à la propriété dans des conditions très avantageuses. A la veille du changement politique de 1989, sur l'ensemble des logements rénovés, $56 \%$ appartenaient désormais aux offices locaux de HLM, contre $6 \%$ avant rénovation, $6 \%$ seulement appartenaient encore à la Ville contre $40 \%$ auparavant, $37 \%$ appartenaient à des propriétaires privés contre $54 \%$ auparavant. (Cf. « Dossier de DSQ, Mairie d'Amiens, $1989 »)$. D'après estimation du recensement 1990, le quartier est passé de 2200 habitants en 1982 à environ $3500^{5}$. Sur cet ensemble $50 \%$ représentaient des anciens du quartier relogés, pas obligatoirement dans le même logement, après une étape courte en logement-tiroir ou de plusieurs années en HLM hors quartier, $15 \%$ étaient encore dans du non rénové. Les $35 \%$ restants correspondaient à des nou veaux résidents dont le profil sociologique était assez typé, il s'agissait de ménages appartenant surtout aux classes moyennes salariées. D'autres étaient les attributaires de logements en tant que " nouveaux commerçants » de boutiques récentes ouvertes à l'initiative du maître d'ouvrage.

3. Il est impossible d'être plus précis car à partir du recensement 1990 l'INSEE, sur recommandation de la CNIL, a procédé à des regroupements pour les quartiers inférieurs à 5000 habitants. Les résultats concernant Saint-leu ont ainsi été regroupés avec ceux concernant le quartier « Palais de Justice », limitrophe mais d'un type urbain totalement différent (bourgeois ancien). Ce chiffre représente une estimation par soustraction du chiffre de population du quartier « Palais de Justice » 1982 de celui concernant le nouveau quartier INSEE St-Leu + "Palais de Justice » 1990. Ce calcul approximatif peut être considéré comme ayant une certaine pertinence du fait de la grande stabilité du quartier "Palais de Justice » (stabilité estimée à partir de celle d'un autre quartier limitrophe de même type bourgeois ancien-supérieur à 5000 habitants, donc recensé de façon identique en 1982 et 1990 et qui n'a pas vu sa population varier entre ces deux dates). 


\section{DES STRATÉGIES D'ACTEURS}

\section{1. ÉVOLUTIONS DES SYSTĖMES DE PRATIQUES ET REDÉFINITIONS D'IDENTITÉS}

Ainsi que Coing (1966) l'avait déjà été constaté, l'entrée dans le nouvel habitat a modifié les systèmes de consommation, mais, et contrairement à ce qui semblait s'être passé dans le cas du treizième arrondissement de Paris, ces changements ici ne sont pas apparus comme jouant un rôle compensatoire par rapport à la perte de l'ancienne vie de quartier. Si celle-ci a évolué, elle a néanmoins conservé beaucoup de ses anciennes caractéristiques. Ce qui a changé en premier lieu ce sont les organisations des budgets familiaux ${ }^{6}$. Les frais relatifs aux logements sont désormais plus élevés malgré la relativement bonne compensation que constituent les APL. Le confort nouveau entraîne des charges de chauffage et cau chaude supéricures aux anciennes. D'autres postes budgétaires peuvent aussi être nouveaux comme le téléphone. Ces charges fixes plus élevées - les loyers antéricurs dépassaient rarement la centaine de francs - requièrent des budgets gérés de façon plus prévisionnelle qu'auparavant où les dépenses ćtaient la plupart du temps engagées au fur et à mesure du besoin, selon un modèle déjà analysé dans d'autres contextes de quartiers urbains pauvres (Lewis, 1963).

Les modes d'approvisionnement ont été aussi modifiés par la disparition des petits commerces locaux, nombreux auparavant. La fréquentation des supermarchés s'est diffusée exigeant aussi des types de gestion financière différents. Cependant les réseaux communautaires antérieurs ont pu être utilisés efficacement. Les courses sont dorénavant souvent faites collectivement dans les grandes surfaces de périphérie, et ceux qui ont des voitures emmènent ceux qui n'en ont pas. Les plus jeunes ramènent des provisions aux plus âgés. Les nouveaux postes budgétaires comme les transformations de consommation entraînées par l'entrée dans le nouvel habitat ne semblent pas avoir eu pour conséquence des phénomènes de surendettement ainsi que l'avaient craint les travailleurs sociaux qui ont suivi les dossiers de relogement. Malgré leurs faibles niveaux de revenus ces familles ont dans la plupart des cas bien contrôlé le changement de modèle. Elles n'ont par exemple acheté que peu de nouveaux meubles, à peu près pas eu recours à des crédits contrairement à ce qui se passe fréquemment après un emménagement ${ }^{7}$.

Le choix pour le relogement sur place a pu sélectionner parmi l'ancienne population les revenus les plus réguliers, néanmoins ce seul facteur n'épuise pas la logique de ces relogements. Les savoir-faire et habitudes antéricurs concernant la sphère économique ont pu être aussi utilisés. Le chômage au moment de l'enquête était toujours aussi important que dix ans auparavant $(20 \%)$ - alors qu'au niveau de l'agglomération il avait cru de plusieurs points - et le travail au noir, parfois très " qualifié ", s'y était quasi intitutionnalisé, alimenté par les réhabilitations menées de façon privée, mais aussi par certaines des opérations municipales où les accédants auto-réhabilitants préféraient payer la compétence d'un voisin. Des spécialités ont ainsi émergé : les installations de chauffage sont faites par un actuel employé

6. Estimés par questionnaire au cours des enquêtes à domicile cf. supra, note méthodologique.

7. Et ainsi que l'avait noté Coing (1966): "En arrivant dans une maison neuve, il faut l' habiller entièrement à ses goûts, la refaire pour soi. ». Ici apparemment les habitants n'avaient pas cu à se « réapproprier » l'espace. 
municipal, ancien chauffagiste, la menuiseric, installation de placards et de cuisines "intégrées » par un chômeur et ainsi de suite. Plusieurs familles ayant désormais un logement plus grand qu'auparavant (en fonction des normes ouvrant droit aux APL, ou mêmes récents accédants) ont loué ou sous-loué des chambres à des étudiants. Des femmes au foyer se sont mises à proposer des « tables d'hôtes » à ces étudiants. D'autres ont pris des enfants en garde pendant la journée, de façon déclarée ou au noir. De tout temps les habitants du quartier étaient assez nombreux à s'occuper d'un jardin potager hors du quartier. Ils sont nombreux à avoir maintenu cette pratique. Certains vendent quelques produits maraîchers et les clients ne manquent pas dans le voisinage. Ces ressources supplémentaires, liées à la fois à la centralité du quartier et à sa composition sociologique plus diversifiée, permettent la plupart du temps de faire face aux nouveaux frais. Le chômage a ici depuis longtemps fait partie du paysage comme est aussi depuis longtemps banalisée la gestion de l'alternance ou du cumul, travail salarié, travail au noir, chômage, ressources épisodiques. On trouve même parmi ceux qui ont récemment emménagé un certain nombre de Rmistes, complétant ce revenu par du travail au noir.

Parmi les changements de pratiques entraînés par l'entrée dans le nouvel habitat, il y a aussi certainement une plus grande polarisation sur le foyer, plus confortable, qui peut s'être traduite par un certain repli sur l'espace privé. Les responsables associatifs se plaignent de cet état de fait. Assez âgés pour la plupart, ils ont du mal a trouver des remplaçants. C'est peut-être aussi l'alternative communautaire que constituait la structure associative traditionnelle qui a perdu son caractère de nécessité. Certaines habitudes demeurent cependant comme les quêtes en cas de deuil et le " colis des vieux » à Noël, pratiques ordinaires d'une entraide populaire mais à peu près disparues aujourd'hui des cités modernes (Schwartz, 1990) et auxquelles les nouveaux résidents s'associent. Ce recul d'audience des anciennes associations correspond peut-être sculement à leur redéfinition. Certaines sont demeurées pleinement actives (sport), d'autres se sont reconstruites soit sur des bases pleinement nouvelles soit en liaison avec des associations à l'initiative de nouveaux habitants (théâtre). Dans ce secteur en recomposition, et dans les deux types d'associations (anciens et nouveaux), l'enjeu semble être l'ouverture à l'autre population, gage souvent de l'obtention de subventions, mais aussi vecteur des nouvelles identités locales pour les uns comme pour les autres.

Malgré ce relatif recentrement sur l'espace privé, la vie de quartier et certains modes d'exercice de la citoyenneté locale, voire même une certaine capacité d'action collective, sont loin d'avoir disparu. En témoignent deux luttes ponctuelles engagées contre la Municipalité d'après 1989 à propos du réaménagement du parc public contigu au quartier. La première a été menéc lorsqu'il fut envisagé de transformer le terrain de «foot » en « terrain de sport polyvalent avec buts amovibles» (sic). À force de manifestations, distributions de tracts, campagnes de signatures et démarches multiples, les jeunes et les responsables associatifs obtinrent la promesse de la conservation d'un terrain ad hoc et même de surcroît celle de la construction d'un vestiaire « en dur ». La seconde fut engagée pour contrer la destruction prévue de quelques pavillons bordant le chemin de hallage en lisière du parc, en bon état mais construits dans les années cinquante selon les canons de l'esthétique populaire et ne correspondant pas à l'esprit du projet. Réunis en association les habitants des maisons concernées parvinrent à mobiliser l'ensemble de la population y compris 
les nouveaux résidents ainsi que la presse locale et ne désarmèrent que lorsque les responsables locaux revinrent sur leur décision d'expulsion. Ceux-ci se montrèrent surpris par une combativité qu'ils n'imaginaient pas pouvoir être le fait d'une population perçue par eux comme culturellement démunie.

Un autre domaine des vies quotidiennes semble avoir été atteint, de façon indirecte mais significative, par l'entrée dans le nouvel habitat et la transformation du quartier. Il s'agit de mobilisations familiales plus fortes autour des scolarités des enfants. L'école est un thème important des discours. Beaucoup évoquent même que ce souci est nouveau et qu'ils ne faisaient pas aussi attention « avant », par exemple pour les plus âgés de leur progéniture. Ces discours se traduisent aussi par des pratiques nouvelles comme l'adhésion à des associations de parents d'élèves, des éventuels changements d'école, le recours au privé etc... autant de comportements dénotant des stratégies d'écoles, possibles dans cette partie du centre-ville où l'offre d'équipements scolaires est importante, qui ne participaient pas auparavant des habitus du quartier. Il peut y avoir là un effet global de génération scolaire, à savoir qu'il y a de façon générale dans l'ensemble des catégories sociales une attention croissante portée à la scolarité. Mais des enquêtes menćes dans le cadre d'une autre recherche, faites à la même époque dans la même agglomération, parmi les mêmes catégories sociales, et en ZUP, n'avaient pas révélé cela dans les mêmes proportions. Cette tendance a été si marquée que l'école primaire du quarticr, dont la réputation était mauvaise, a été peu à peu vidée d'une part importante de ses effectifs. N'y restèrent que des enfants appartenant à des familles à graves problèmes sociaux. Plusieurs épisodes de violence et de dégradation conduisirent à sa fermeture " pour travaux » et à la dispersion de l'effectif restant dans les écoles environnantes.

On peut sans doute expliquer cette évolution du rapport à l'école de plusieurs façons ou par la combinaison de plusicurs facteurs. Des logements plus grands offrant presqu'une chambre par enfant peuvent entraîner un rapport à eux plus individualisé. Ce type d'interactions entre habitat et pratiques a déjà été souligné (Haumont, 1968). On peut aussi imaginer devoir prendre en compte une auto-sélection de la population ayant opté pour un logement rénové et plus cher dans le quartier avec un projet de sortic de "risque de précarisation ", la mobilisation scolaire pouvant alors appuyer ce projet. Le déclin social qui avait marqué le quartier dans les dernières déce inies d'avant la rénovation hante encore beaucoup de discours. Enfin entre aussi en ligne de compte un facteur que l'on pourrait qualifier d' "effet de milieu » 8 , et qui cette fois ci n'est pas verbalisé par les familles en tant que tel, mais observé et interprété par nous. La cohabitation dans certains secteurs du quartier entre " anciens " et « nouvcaux » a donné licu à des rapports classiques de voisinage de type lotissement pavillonnaire qui se traduisent surtout par des « copinages » d'enfants. De multiples petites anecdotes témoignent de cet effet de milieu. Ainsi cette mère de famille originaire du quartier qui dit que c'est sur les conseils de sa nouvelle voisine enseignante qu'elle a changé son fils d'école afin qu'il soit " plus encadré ». Leurs enfants sont du même âge, se reçoivent mutuellement le mercredi et font leur travail ensemble. Même si ces anecdotes ne

- "L'origine première de tout processus social de quelque importance doit être recherchée dans la constitution du milieu social interne (Durkhcim, 1960). 
relèvent que du déclaratif, elles témoignent d'un changement de mentalité qui, lui, peut être important 9 .

\section{2. MOBILISATION DE RESSOURCES ET RECONVERSION DE CAPITAUX}

Si nous avons souligné ce qui dans les vies matérielles, les pratiques quotidiennes et certaines attitudes des anciens ménages du quartier nous paraissait avoir été modifié par l'entrée dans le nouvel habitat, nous avons pu saisir aussi, et le type d'approche retenu l'a permis, que c'était plus que cela qui avait été en jeu dans ce « choix »d'être resté dans le quartier. Loin d'avoir été complètement dominés par des événements extérieurs à eux - la rénovation - les uns et les autres de ces ménages, pas tous avec la même opportunité matérielle et culturelle ni les mêmes chances de réussite, ont pu mener d'une certaine manière des stratégies sociales, dont les enjeux émergeront progressivement. Celles-ci n'étaient pas forcément conscientes en tant que telles mais peuvent au bout du compte s'analyser ainsi. « La notion de stratégie est l'instrument d'une rupture avec le point de vue objectiviste et avec l'action sans agent que suppose le structuralisme (en recourant par exemple à la notion d'inconscient). Mais on peut refuser de voir dans la stratégie le produit d'un programme inconscient sans en faire le produit d'un calcul conscient et rationnel » (Bourdieu, 1987). La présentation de trois cas résumés va tenter de faire comprendre cela. Les deux premiers ménages ont été retenus en raison de leurs caractéristiques proches (même âge, même type de ménage avec femmes au foyer et nombreux enfants, maris employés du secteur public mais selon un statut plus stable pour le premier que pour le second) et de leur intégration différente de l'épisode rénovation. Le troisième cas, une vieille dame, veuve, qui a travaillé la plus grande partie de sa vie et élevé beaucoup enfants, est à la fois représentatif de l'histoire du quartier et du déblocage des histoires familiales auquel la rénovation a pu contribuer.

- Les T. ont respectivement 45 et 49 ans. A eux deux, de plusieurs unions, ils ont eu douze enfants dont cinq sont encore à la maison. M. T. est postier et sa femme n'a jamais eu d'activité professionnelle. Lui est venu enfant dans le quartier et y a presque toujours résidé depuis. Ses parents ouvriers ruraux avaient migré vers la ville. Elle, dont les parents étaient petits commerçants dans un faubourg ouvrier voisin, ne réside dans le quartier « que depuis 30 ans ». Trois de leurs enfants adultes sont « mariés dans le quartier ». Ils habitaient auparavant en location dans une vieille maison appartenant à la Ville. Après avoir longuement hésité et fait leurs comptes ils ont opté pour l'accession à la propriété en auto-réhabilitaiton, rachetant par la même occasion et selon la même formule la maison voisine à fin d'agrandissement.

- Si la sociabilité de voisinage demeure limitée entre anciens et nouveaux résidents et n'a rien à voir avec celle qui concemait les anciens, elle n'est pas inexistante. Les constatations du maintien d'une distance sociale malgré une proximité spatiale, faites dans un article devenu classique (Chamboredon, Lemaire, 1970) correspondaient au contexte spécifique des grands ensembles de cette époque où résidait une relative diversité de couches sociales, moyennes et populaires, les unes et les autres n'étant pas dans la même position sur leur trajectoire résidenticlle. Il est vraisemblable que dans le cas présent, l'antériorité de résidence, la supériorité numérique et la forte identité sociale liée au quartier, qui sont les attributs des couches social es populaires, aient permis une certaine atténuation de la distance sociale, autorisant certaines formes de relations de voisinage, distance qui aurait pu être insurmontable dans une aut re situation socio-spatiale. 
Le faible prix de base, un prêt PAP plus une bonne APL leur autorisaient des traites qu'ils ont jugées supportables. On leur a également fourni la base des matériaux pour les travaux. Ils ont même revendu une partie de ceux-ci pour en racheter d'autres plus à leur convenance. Il se sont fait aider des années durant par un beau-frère maçon. Depuis la fin des travaux, Mme $T$. a « pris des pensionnaires », il s'agit d'étudiants qu'elle loge et nourrit. Alors que les aînés de leurs enfants « ont pas été longtemps à l'école » et qu'ils " ne savent d'ailleurs pas pourquoi ", ils ont mis les deux derniers à l'école privée. «C'est pas par snobisme » précise-t-elle « mais on les aide mieux pour les devoirs, et puis aussi ils traînent pas parce qu'ils les gardent tard. » Elle dit assister désormais à toutes les réunions de parents d'élèves, ce qu'elle n'avait jamais fait auparavant.

Dans leur rue, plusicurs nouvelles familles sont arrivées. "A côté, c'est un médecin, j'ai été me présenter, voir s'il avait besoin de rien, comme il connaissait pas... Moi à sa place je me serais sentie expatriée d'arriver dans un quartier qui n'était pas le mien... ». L'un et l'autre estiment que « c'est plutôt bien d'avoir comme maintenant une population hétéroclite »10. M. T. milite toujours comme avant à son travail et dans une association sportive locale. Leur vie de quartier est toujours la même « avec quelques nouveaux voisins en plus, c'est un peu une ambiance nouvelle aussi peut-être ». Les T. ćtaient auparavant intégrés économiquement et socialement mais selon une citoyenneté locale enfermante du point de vue de la mobilité sociale. La rénovation leur a permis l'accession à la propriété en centre-ville qui n'aurait jamais été imaginable dans un autre contexte. Mais clle a aussi été possible parce qu'ils en ont eu les capacités culturelles. Cette propriété acquise aussi en partic grâce aux ressources supplémentaires liées à sa centralité les fait accéder à un statut social amélioré. Les T. n'ont pas de fragilité de statut, ni de carence d'identité sociale. Ils sont pleinement intégrés dans le travail et la société de voisinage. Lui comme militant CFDT exprime une vision du monde qui valorise le consensus social et le mélange des classes. Ce système de représentations ne leur fait pas percevoir l'arrivée des nouveaux habitants comme une invasion ou une agression. Au contraire, ils vont retourner à leur avantage cette transformation sociologique du quartier. Celte espèce d'aisance sociale et mentale leur permet de mobiliser leurs énergies sur l'économique - pour réussir leur accession à la propriété - et sur l'avenir scolaire de leurs derniers enfants qui constitue de façon nouvelle pour eux un objectif relativement clair.

- Les M. ont le même âga que le couple précédent, 45 et 47 ans. Ils ont sept enfants dont quatre encore présents. Ils appartiennent l'un et l'autre à des " grandes familles » présentes dans le quartier depuis des générations. Leurs pères étaient ouvriers dans les usines textiles. Il est employé municipal, et vient juste de retrouver du travail après deux ans de chômage. Durant cette période il "s'en tirait quand même bien », dit-il, parce qu'il « avait beaucoup de chantiers ». Sa femme n'a jamais exercé d'activité professionnelle. Ils louent une maison rénovée de cinq pièces pour $3000 \mathrm{~F}$ par mois presqu'entièrement couverts par l'APL « mais dans quatre ans j'en aurai plus et puis de toutes les façons c'est sûr qu'ils vont augmenter les loyers le

10. Une précédente étude (Pétonnet, 1982) constatait que dans les quartiers ouvriers en rénovation, «les anciens habitants qui ne supportent pas le nouveau voisinage quittent les lieux accélérant ainsi le processus de renouvellement". Ici cette tendance à une perception \& d'un quartier qui a perdu son âme » (cf. plus loin), a été contrée par un processus inverse, le nouveau voi sinage a été perçu par certains comme opportunité de déstigmatisation. 
nouvel Office, c'est ça sa politique, faire un quartier de rupins ». M. a toujours ćté militant politique et leader associatif. Il a entrepris de monter une nouvelle formule associative, (loisirs pour les enfants et animation de quartier) où il essaye « d'attirer des nouveaux résidents, parce que c'est ça qui est intéressant ».

Mais malgré tout $M$. a du mal à accepter la transformation du quartier qui selon lui « a perdu son âme ». Il apparaît à certains moments aigri et nostalgique, tout en tentant des ouvertures vers la nouvelle population. Autrefois figure archétypale du quartier, il en était aussi une personnalité centrale. "Paresseux et grande gueule mais génércux et débrouillard » dit de lui un membre de sa famille étendue. N'ayant jamais eu une grande stabilité professionnelle, c'est autour de son statut dans le quartier qu'il avait construit son identité. Or il se sent fragilisé dans ce nouvel espace, bien qu'il y tente une reconversion identitaire et statutaire. Il se sent aussi fragilisé par rapport à son logement. Il a très peur de devoir quitter le quartier en raison des hausses de loyer qu'il prévoit. Peut-être pourra-t-il y trouver un logement plus petit et moins cher? Il est en pleine phase de reconstruction de son système de représentations. Parfois il exprime une représentation dualiste et conflictuelle d'un monde social clivé entre « les rupins » (« eux », « tous les autres ») et les « pauvres » (« nous »). Par contre, dans ses nouvelles fonctions associatives, c'est le mélange social qu'il essaye de promouvoir et de construire. Dans cette période d'incertitudes, il a du mal aussi à se projeter à travers ses enfants. Il craint pour certains, dit-il, qu'à leurs dix-huit ans, ils se mettent à voter FN. « Pour un vieux communiste comme moi ça fait plutôt mal au coeur », ajoute-t-il. Si M. ne parvient pas à rester dans le quartier qui était, plus encore que le cas précédent (postier), son principal vecteur d'inscription sociale, et où il fait des efforts de reconstruction identitaire, on peut penser qu'il peut glisser vers le « cas social ». Les $M$. doivent se recomposer des représentations du monde, du quartier et d'eux-mêmes cohérentes, travail que la famille précédente n'avait pas à accomplir. Les M. ne sont de ce fait pas encore en état de se mobiliser comme les $\mathrm{T}$. pour une amélioration ćconomique de leur situation ni de formuler des projets bien définis à propos de leurs enfants. Ils y parviendront peut-être dans un deuxième temps. Mais les enfants auront grandi.

- Mme B. a soixante-dix ans. Elle est originaire du quartier où elle a résidé toute sa vie presque sans interruption et où elle appartient aussi à une des « grandes familles » locales. Ses parents étaient ouvriers-tisseurs, ceux de son mari commerçants-ambulants. Elle-même a été ouvrière en filature lorsqu'elle était jeune puis plus tard femme de service à l'hôpital. Son mari d'abord commerçant est devenu ensuite électricien et est mort depuis vingt ans. Ils « n'ont jamais été au chômage et ont toujours bien gagné leur vie », dit-elle. Ils ont eu huit enfants qu'elle a fini d'élever seule. Elle vit actuellement avec une de ses petites filles de vingt ans et l'enfant de celle-ci. Elles sont locataires d'une petite maison dont le loyer est bien couvert par leur APL. Sa retraite et les prestations sociales leur permettent de vivre selon elles « très correctement ». Une de ses filles vit à proximité. A l'écouter raconter l'histoire de la famille, on saisit à la fois tout le contexte temporel global où la régularité des salaires avait assuré une amélioration régulière des niveaux de vie, mais aussi comment le contexte local avait influé de façon différente sur le déroulement des vies.

Quatre de ses enfants « ont maintenant des belles situations » dit-elle, tandis que des quatre autres, l'un a été tué dans un règlement de compte local (par le fils d'une 
autre famille du quartier) et trois ne donnent plus de nouvelles. Ils « habitent dans le sud (de la France) mais ils doivent pas se rappeler qu'ils ont une mère » dit-elle avec amertume, ajoutant, " ceux-là ils en ont fait des bêtises ! ». A l'heure actuelle on a l'impression que le quartier rénové l'aide un peu à effacer le contexte du drame. Elle fait constamment des rapprochements d'images, laissant à penser que le confort et la propreté sont à l'image des vies nouvelles qui vont pouvoir s'y dérouler. « S'ils avaient pas eu des mauvaises influences mes fils, ça se serait jamais passé comme ça ». Elle évoque avec fierté certains de ses " petits enfants qui sont étudiants et travaillent très bien ». Elle développe par elle-même, sur un ton de rancoeur parfois de colère, toute une analyse de l'enfermement dans la pauvreté que le quartier a connu, dans lequel elle voit la cause orignelle des «bêtises » de ses fils, et que la rénovation, elle l'espère, va faire oublier. Comme si elle tentait de récupérer une identité qui aurait dû toujours être la sienne, celle d'une condition ouvrière faite de vie matérielle " aisée » et de "vie droite ». Elle semble avoir « récupéré » des conditions de vie qui la rétablissent dans les représentations qu'elle avait d'elle-même.

On saisit à travers ces cas comment la rénovation peut à la fois avoir déstabilisé une population qui tirait l'essenticl de son identité d'un territoire traditionnel paupérisé, mais comment aussi dans le même temps elle peut l'avoir désenclavée culturellement. Cependant dans les trois cas le relogement s'est traduit par une ouverture du choix des possibles par rapport à leur condition sociale antérieure. La rénovation aura joué potentiellement comme un opérateur de rétablissement social. Il est en train de s'y jouer un processus contraire à ce qui s'y était passé il y a trente ans. Alors que le contexte global était à l'ouverture des trajectoires sociales, celles des habitants du quartier avaient en général été dans le sens de la stagnation ou du déclin. Dans le contexte actuel caractérisé plutôt par des itinéraires de stagnation ou de déclin, ces familles paraissent en mesure de se réapproprier des potentialités d'ouverture sociale. Mais si cela a été possible, c'est parce que le changement social résultant de l'événement rénovation, a été intégré, bien que pas par tous avec la même aisance. Et cette intégration est liće, on peut en faire tout au moins l'hypothèse, à un "effet-territoire », c'est-à-dire à la prégnance de cette culture populaire territorialisée. Des agents sociaux, largement dominés dans la structure sociale globale, ont pu agir en acteurs, constituant en capital leurs ressources. Ils ont construit leurs stratégies à partir de ce qui ćtait à leur disposition, en l'occurrence un certain type de capital, culturel, social, informationnel, de caractère spécifique car à usage et done à valeur presqu'exclusivement interne (au quartier). Dans le cas des T. par exemple, ce capital informationnel leur a permis d'être dans les bons circuits, d'être bien conseillés pour monter leur dossier d' "accession ». Dans leurs pratiques actuelles, ils essayent de reconvertir un capital à strict usage interne en un capital à valeur plus générale. Leur aspiration à intégrer les règles du jeu scolaire peut s'analyser comme la tentative de dépasser la règle du jeu locale pour s'inscrire dans un « nomos » (au double sens grec de « loi » et de « district») plus général. Et le " scolaire " peut constituer une voie d'accès au général ainsi que le notait déjà Weber (1971) : "Le titre scolaire a pour fonction de produire des individus interchangeables ». Dans le second cas, M. essaye de reconvertir son capital social antérieur (personnalité du quartier traditionnel) en un capital social à plus large 
spectre, cherchant la reconnaissance de sa compétence de militant associatif auprès de nouveaux acteurs sociaux.

\section{3. EXCLUSION SOCIALLE ET * REQUALIFICATION TERRITORIAIE *}

Cette enquête a été menée deux ans seulement après le changement de Municipalité, lorsque l'inflexion qui semblait s'amorcer dans les politiques urbaines de l'ensemble de l'agglomération et en particulier dans celles concernant l'achèvement de cette opération ne s'était pas encore fait sentir 11. Il semblait néanmoins évident que le caractère social qu'elle avait eu risquait d'être mis à mal, en particulier à travers une révision de loyers que pouvait mettre en place l'Office HLM dès qu'il le pourrait, les procédures de financement utilisées ayant imposé leur blocage sur dix ans. C'est aussi tout ce qu'il restait à rénover, " privatisé » après le changement politique, qui a vu son statut et sa population rapidement changer depuis. Il serait regrettable que toutes les dynamiques sociales qui s'étaient progressivement mises en place sur une dizaine d'années soient remises en question. Si le quartier de Saint-Leu dans les années quatre-vingt avait été finalement rasé puis rénové « classiquement », sa population éparpillée aurait perdu le rapport particulier qu'elle avait entretenu avec son territoire, comme les ressources socio-culturelles qu'elle avait pu y capitaliser. Il est probable que la plupart des ménages auraient alimenté l'aggravation des problèmes d'exclusion socio-économique qu'ont connu depuis les quartiers périphériques de cette ville. La rénovation de ce quartier telle qu'elle a été menée a constitué, avant que le thème n'émerge dans le discours public, une politique efficace d'intégration et de lutte contre l'exclusion. Appliquée à une population qui avait des caractéristiques sociales et culturelles particulières, elle aura sans doute permis la réintégration dans des trajectoires sociales potentiellement plus ouvertes à la mobilité des ménages qui, dans un contexte particulier, en avaient été exclus.

Les politiques dites de la Ville menées par le gouvernement socialiste de l'époque comme celles que semblent aussi adopter l'actuel, dont l'un des porte-parole en la matière est précisément le premier magistrat de cette ville, ont pour objectif déclaré de mettre en place des procédures de "lutte contre l'exclusion » (Donzelot, 1991, 1994), en oeuvrant à la « requalification territoriale » ainsi qu'à la " recréation du lien social », dans les espaces listés comme quartiers ou banlieues « difficiles » et dont trois ZUP d'Amiens font d'ailleurs partie. Dans le même temps les processus de constitution et de peuplement de ces espaces ne sont jamais interrogés. Les effets de ségrégation découlant des « modernisations » des centres-ville telles qu'elles y sont menées par les décideurs locaux ne sont jamais remis en cause. Dans le cas qui nous concerne, c'est presque de toutes pièces que des difficultés socio-économiques jusque là contenues et auto-gérées risquent d'être construites en «problèmes sociaux ». Ceux-ci seront alors «traités » par ces dispositifs financés par l'État.

1. Il est rare que sur le long terme des telles opérations ne connaissent pas de changements de trajectoires par l'entrée en jeu de nouveaux acteurs, ainsi que l'a noté Marié (1970). 


\section{REFERENCES BIBHOGRAPHIQUES}

BIDOU-ZACHARIASEN, C. (avec la collaboration de LEPRESLE, M.). Rénovation sociale et valorisation culturelle d'un quartier historique, Amiens face à son patrimoine. Rapport IRIS, 1993.

BLANC, M. De l'habitat insalubre au logement social dévalorisé. Les Annales de la Recherche urbaine, décembre 1990, n`49, p. 37-48.

BOURDIEU, P. Choses dites. Paris, Editions de Minuit, 1987, p. 79.

CHAMBOREDON, J.-C., LEMAIRE, J. Proximité spatiale et distance sociale. Revue Française de Sociologie, 1970, vol. XI, $n^{\circ} 1$, p. 3-33.

COING, H. Rénovation urbaine et changement social. Paris, Les Editions ouvrières, 1966, p. 14.

DEBONNEUIL, X., GOLAC, M. Structure sociale des villes. Economie et Statistique, mars $1978, n^{\circ} 98$, p. 51-65.

DURKHEIM, E. Les Règles de la méthode sociologique. Paris, PUF, 1960, p. 111.

DONZELOT, J. (ed). Face a l' exclusion, le modele français. Paris, Editions Esprit, 1991. DONZELOT, J. L'Etat médiateur. Paris, Editions Esprit, 1994.

GURVITCH, G. La vocation actuelle de la Sociologie, tome I. Paris, PUF, 1963, p. 161.

HARRISON, P. Inside the inner cities. Londres, Harmondsworth, Penguin Book, 1983.

HAUMONT, N. Habitat et modèles culturels. Revue Française de Sociologie, 1968, vol. IX, $n^{\circ} 2$, p. 180-190.

HOGGART, R. La Culture du pauvre. Paris, Editions de Minuit, 1970.

LEFEB VRE, H. Le Droit a la Ville. Paris, Editions Anthropos, 1968, p. 65.

LEWIS, O. Les enfants de Sanchez. Paris, Editons Gallimard, 1963.

MARIÉ, M. Sociologie d'une rénovation urbaine. Sociologie du Travail, $1970, \mathbf{n}^{\circ} 4$, p. 469-488.

PÉTONNET, C. Espaces habités, ethnologie des banlieues. Paris, Galiléc, 1982, p. 27.

PLAN CONSTRUCTION. Typologies de l'llabitat ancien (1850-1948). Actes de Colloques, 1979.

SEGALEN, M. Nanterriens, les familles dans la ville, une ethnologie de l'identité. Toulouse, Presses Universitaires du Mirail, 1990.

SÉLIM, M. Rapports sociaux dans un quartier anciennement industriel, un isolat social. L'Homme, octobre-décembre 1982, Tome XXII, n`4, p. 77-86.

SCHWARTZ, O. Le monde privé des ouvriers, hommes et femmes du Nord. Paris, PUF, 1990.

VERRET, M. L'espace ouvrier. Paris, Armand Colin, 1979.

WEBER, M. Economie el société. Paris, Plon, 1971.

WILLMOTT, P., YOUNG, M. Family and kindship in East London. Londres. Londres, Routledge and Kegan, 1957. Traduction française, Le village dans la ville. Paris, Editions du CCI, 1983. 